\title{
Effects of Xuezhikang vs. Pravastatin on
}

Triglyceride Level in Patients with Type 2 Diabetes

Mellitus and Dyslipidemia: A Research Protocol for a Randomized Controlled Study

Jin Xu

Second Xiangya Hospital

Xiao Du

Second Xiangya Hospital

Shilan Zhang

Second Xiangya Hospital

Qunyan Xiang

Second Xiangya Hospital

Liling Guo

Second Xiangya Hospital

Liyuan Zhu

Second Xiangya Hospital

Yangrong Tan

Second Xiangya Hospital

Yan Fu

Second Xiangya Hospital

Yingying Xie

Second Xiangya Hospital

Miao Zhang

Second Xiangya Hospital

Tie Wen

Second Xiangya Hospital

Ling Liu ( $\nabla$ feliuling@csu.edu.cn )

Second Xiangya Hospital https://orcid.org/0000-0001-6979-701X

\section{Research}

Keywords: diabetes, triglyceride, dyslipidemia, Xuezhikang, Cardiovascular risk

Posted Date: September 14th, 2021 
DOI: https://doi.org/10.21203/rs.3.rs-871652/v1

License: (c) (1) This work is licensed under a Creative Commons Attribution 4.0 International License. Read Full License 
$1 \quad$ Effects of Xuezhikang vs. Pravastatin on triglyceride level in

2 patients with Type 2 Diabetes Mellitus and dyslipidemia: a

3 research protocol for a randomized controlled study

4 Jin $\mathrm{Xu}^{1,2,3,4}$, Xiao $\mathrm{Du}^{1,2,3,4}$, Shilan Zhang ${ }^{1,2,3,4}$, Qunyan Xiang ${ }^{1,2,3,4}$, Liling Guo ${ }^{1,2,3,4}$,

5 Liyuan $\mathrm{Zhu}^{1,2,3,4}$, Yangrong Tan ${ }^{1,2,3,4}$, Yan $\mathrm{Fu}^{1,2,3,4}$, Yingying $\mathrm{Xie}^{1,2,3,4}$, Miao Zhang ${ }^{1,2,3,4}$,

6 Tie Wen ${ }^{5,6}$, Ling Liu ${ }^{1,2,3,4 *}$

7 1. Department of Cardiovascular Medicine, the Second Xiangya Hospital, Central $8 \quad$ South University;

9 2. Research Institute of Blood Lipid and Atherosclerosis, Central South University;

3. Modern Cardiovascular Disease Clinical Technology Research Center of Hunan Province;

* Address correspondence to this author at: \#139 Middle Renmin Road, Changsha, Hunan 410011, PR China. Fax +86-073185295407; E-mail: feliuling@csu.edu.cn. 


\section{$1 \quad$ Abstract}

2 Background: Diabetes often accompanies with increase in triglyceride(TG) as well 3 as small dense low density lipoprotein(sdLDL). Statins are difficult to completely 4 correct this dyslipidemia. Xuezhikang, an extract of cholestin, is better than some 5 statins in reducing TG. Under the condition of the similar decrease in 6 LDL-cholesterol (LDL-C), it is not clear whether there is any difference in the effect 7 of XZK and statins on TG reduction in patients with Type 2 DM(T2DM).

8 Methods: An open-label multicenter study is planned to estimate the effects of 9 Xuezhikang(1.2 g/d) and pravastatin(20 mg/d) on TG level and other blood lipids in 10 T2DM patients with dyslipidemia and moderate to high risk of cardiovascular 11 diseases. A total of 114 patients will be enrolled and randomly assigned (1:1 ratio) to accept Xuezhikang or pravastatin therapy for 6 weeks. The primary outcome measure is the change in fasting TG level after 6 weeks. The changes in other fasting lipids and postprandial lipids at 1, 2, $4 \mathrm{~h}$ after a nutritious breakfast will also be explored. The planned duration for enrollment is between November 2021 and December 2022.

Conclusion: This study will evaluate the effect of 6-week treatment of Xuezhikang(1.2 g/d) and pravastatin(20 mg/d) on fasting TG level and other blood lipids in T2DM patients with dyslipidemia, which may provide a more optimized schedule for lipid control in patients with diabetes and dyslipidemia in primary prevention.

Keyword: diabetes; triglyceride; dyslipidemia; Xuezhikang; Cardiovascular risk 


\section{Background}

Type 2 diabetes mellitus(T2DM) obviously increases the risk of atherosclerotic cardiovascular disease (ASCVD)[1, 2], and it is regarded as the equivalent of coronary heart disease. Diabetes often accompanies with a particular dyslipidemia, presenting as decrease in high density lipoprotein cholesterol(HDL-C) and increase in triglyceride(TG) as well as small dense low density lipoprotein(sdLDL)[3]. This makes the blood lipid control of diabetic patients more complicated.

For patients with T2DM, the first goal of lipid-lowering therapy is to control the level of low density lipoprotein cholesterol(LDL-C), no matter in primary or secondary prevention. However, high TG levels in both fasting and non-fasting states also increase ASCVD risk of diabetic patients[4, 5]. Non-HDL-C covers the cholesterol contents in nearly all atherosclerotic lipoproteins, including LDL, lipoprotein(a), TG-rich lipoproteins and their remnants[6]. Thus, non-HDL-C level is recommended for ASCVD risk evaluation, particularly in patients with high TG, diabetes, obesity or very low LDL-C level[7, 8]. Moreover, it is considered that lowering TG level contributes to the reduction of ASCVD risk in diabetic patients.

At present, statins are the cornerstone of cholesterol control in diabetic patients[9]. However, the effects of statins on TG level were quite different in previous studies. For example, rosuvastatin was reported to reduce TG level by $12-37 \%$ across a dose range of 5-40 mg/d[10-12], while pravastatin $40 \mathrm{mg} / \mathrm{d}$ for 12 weeks was found to reduce TG level by $2 \%$ in a multicenter study involved 41 clinical sites in three European countries[13]. Xuezhikang, an extract of cholestin, was reported to achieve greater TG reduction, when it reached equivalent LDL-C reduction as simvastatin[14]. It contains 13 kinds of natural statins, including natural lovastatin and its homologues, as well as unsaturated fatty acids, ergo sterol, flavonoids and other active ingredients[15]. Non-statin elements within Xuezhikang may play a synergistic role in improving blood lipids and inhibiting the synthesis of TG[16]. It was reported that Xuezhikang $1.2 \mathrm{~g} / \mathrm{d}$ and pravastatin $20 \mathrm{mg} / \mathrm{d}$ reduced LDL-C by a similar extent, about $24-28 \%[15,17-20]$. Therefore, on the basis of equivalent reduction in LDL-C level, we conjectured that Xuezhikang $1.2 \mathrm{~g} / \mathrm{d}$ may achieve greater TG reduction than pravastatin $20 \mathrm{mg} / \mathrm{d}$ in $\mathrm{T} 2 \mathrm{DM}$ patients with dyslipidemia . 


\section{Methods and Analysis}

\section{Study design}

This will be an open-label, multicenter, prospective, randomized controlled study to estimate the effects of Xuezhikang(1.2 g/d) and pravastatin(20 mg/d) on TG level and other blood lipid in patients with T2DM and dyslipidemia. The clinical trial registration(trial No: ChiCTR2100046704) can be found at http://www.chictr.org.cn/index.aspx.

Patients with T2DM and dyslipidemia are enrolled and randomly assigned (1:1 ratio) to Xuezhikang(1.2 g/d) or pravastatin(20 mg/d) therapy. Blood lipids at fasting and 1, 2, $4 \mathrm{~h}$ after a nutritious breakfast, will be monitored at baseline and 6 weeks after treatment.

\section{Patient population}

The patients $(\geq 18$ y) with $\mathrm{T} 2 \mathrm{DM}$ and dyslipidemia must meet certain conditions before entering this study. T2DM should be diagnosed according to American Diabetes Association Standards of Medical Care in Diabetes(2020)[21]. Dyslipidemia is diagnosed according to the test within recent 4 weeks, and both of the following conditions should be meet: (1) Fasting TG $\geq 1.7 \mathrm{mmol} / \mathrm{L}$ and $<5.6 \mathrm{mmol} / \mathrm{L}$; (2) Fasting LDL-C $\geq 1.8 \mathrm{mmol} / \mathrm{L}$ and $<4.9 \mathrm{mmol} / \mathrm{L}$. In addition, all enrolled patients should get at least one of the following conditions: (1) T2DM duration $\geq 10$ years; (2) Smoking; (3) Obesity[BMI $\geq 28 \mathrm{Kg} / \mathrm{m}^{2}$, or waist circumference $\geq 90 \mathrm{~cm}$ (male) or $\geq 85 \mathrm{~cm}$ (female)]; (4) Hypertension; (5) Fasting HDL-C $<1.0 \mathrm{mmol} / \mathrm{L}$ or $\mathrm{LDL}-\mathrm{C} \geq 2.6 \mathrm{mmol} / \mathrm{L}$ within recent 4 weeks.

Exclusion criteria are presented as follows:

(1) Subject who meets either of the following (1)-(7) items should be excluded.

(1) Subject with ASCVD including coronary heart disease, ischemic stroke, transient ischemic attacks, peripheral artery disease, etc.

(2) Subject who has taken lipid-lowering drugs in the past three months.

(3) Diabetic patient who has not yet controlled their blood glucose, $\mathrm{HbA1c} \% \geqslant$ $8.0 \%$.

(4) Subject with active liver disease or dysfunction including sustained serum transaminase sustained elevation of unknown origin or higher than 3 times the upper limit of normal .

(5) Subject with myopathy or serum creatine kinase $>5$ times the upper limit of normal not caused by muscle injury. 
(6) Subject with contraindication to Xuezhikang capsules or pravastatin, or long-term taking glucocorticoids or contraceptives.

(7) Subject in the acute stage of various infectious diseases, or with any of the following diseases, such as hyperthyroidism or hypothyroidism, acute cerebrovascular disease, severe cardio-renal insufficiency(glomerular filtration rate $<60$ $\mathrm{ml} / \mathrm{min} / 1.73 \mathrm{~m}^{2}$ ), malignant tumor, hematopoietic disease, autoimmune disease, digestive system disorders affecting digestion and/or absorptive function, mental disorder, severe or unstable physical disease Patients.

(2) Subject who has a history of alcohol or drug abuse or dependence in the past three months should be excluded.

(3) Subject who has participated in clinical trials of other drugs in the past three months should be excluded.

(4) Subject who has other conditions inappropriate for participation at the investigator's discretion should be excluded.

\section{Follow-up}

In this study, the enrolled subjects will go through 4 visits: including initial screening period, baseline period, before and after the 6-week treatment(Figure 1). Subjects agreeing to participate will be invited to a pre-screening that includes evaluating of the study eligibility criteria before being included in the study(visit 1). After screening, eligible subjects will be given a health education and diet direction, and they need maintain it for at least $14 \mathrm{~d}$. No lipid-lowering drug is allowed during this period. After a $14 \mathrm{~d}$ run-in period, subjects will receive detailed blood lipid test and safety assessments, after which ineligible subjects will be excluded(visit 2).

All eligible subjects will be randomized to Xuezhikang group or pravastatin group, and a nutritious breakfast with total calories of $800 \mathrm{kcal}$ will be given as described previously[15], followed by blood lipids test at fasting and 1, 2, $4 \mathrm{~h}$ after the nutritious breakfast(visit 3).

Six weeks after treatment, all subjects will be followed-up for the last time, and fasting and postprandial blood lipids after a $800 \mathrm{kcal}$ breakfast will be measured repeatedly (visit 4).

\section{Outcome measures}

The primary outcome measure is the changes in fasting TG level after 6-week treatment of Xuezhikang $(1.2 \mathrm{~g} / \mathrm{d})$ and $\operatorname{pravastatin}(20 \mathrm{mg} / \mathrm{d})$. While the secondary outcome measure is the changes in other fasting lipid indexes: including total 
1 chelosterol, LDL-C, HDL-C, non-HDL-C, remnant cholesterol, lipoprotein(a), 2 apolipoprotein(Apo) $\mathrm{A} 1$ and ApoB from baseline after 6 weeks treatment. The 3 changes in postprandial TG levels at 1,2 or $4 \mathrm{~h}$ after breakfast from baseline will be 4 explored. And the fasting and postprandial plasma glucose and insulin levels as well as fasting $\mathrm{HbA} 1 \mathrm{c} \%$ level will also be investigated. In addition, the changes in fasting and postprandial C-reactive protein levels will be calculated after 6-week treatment. An overview of performed assessments is provided in Table 1.

\section{Safety}

Safety will be considered throughout the whole study. Adverse events, physical examination, vital signs, resting 12-lead electrocardiogram, blood samples for determination of clinical chemistry(blood routine, creatine kinase, renal and liver function) and urinalysis will be observed.

The principal safety concerns are hepatic dysfunction and myopathy which can be reflected by creatine kinase and liver function analyses. Any adverse events, such as subjective discomfort and abnormal laboratory tests, will be taken seriously, analyzed carefully, and treated immediately to protect the safety of the subjects.

\section{Sample size}

Our primary endpoint is the change in fasting triglyceride level from the baseline. According to the results of Zhao et al. (2003) and Jones et al. (2003), TG decreased by $0.62 \mathrm{mmol} / \mathrm{L}$ from the baseline in the Xuezhikang group, and $0.16 \mathrm{mmol} / \mathrm{L}$ in pravastatin group $[17,22]$. The difference in reduction in fasting TG level after treatment is $0.46 \mathrm{mmol} / \mathrm{L}$, and the standard deviation of mean change in fasting TG level for Xuezhikang group is $0.66 \mathrm{mmol} / \mathrm{L}$ according to previous study[22], while that for pravastatin group is assumed as the same, $0.66 \mathrm{mmol} / \mathrm{L}$. The two-sample t test, with a 0.052 -sided significance level and $90 \%$ power to detect the difference between two groups are used. Approximately 45 subjects per group (or total of 90 subjects) are required, and considering a dropout rate of $20 \%$, a total of 114 patients need to be included in this study. Sample size estimation was performed by a professional statistician using nQuery version 6.01 (Statistical Solutions, Sangus, MA).

\section{Discussion}

There were a variety of studies showed that Xuezhikang capsules significantly reduced both cholesterol level and the incidence of cardiovascular events, as well as the overall mortality in Chinese patients with coronary heart disease[23-25], especially in those with T2DM[23-25]. However, there was no study comparing the 
ability of TG reduction between Xuezhikang, complex of natural statins, and synthetic statins. For subjects with high risk of ASCVD, including diabetic patients without ASCVD and target organ damages but with diabetic duration $\geqslant 10$ years or other additional risk factors, an LDL-C goal of $<1.8 \mathrm{mmol} / \mathrm{L}$ is recommended[26]. Other risk factors refer to age, hypertension, obesity, smoking and dyslipidemia, such as low HDL-C level[26]. It means that even without ASCVD, some diabetic patients with high risk of ASCVD and LDL-C level $\geqslant 1.8 \mathrm{mmol} / \mathrm{L}$ will be enrolled in this study. As for those with moderate risk of ASCVD, including young patients (T1DM <35 years; T2DM $<50$ years) with diabetic duration $<10$ years without other risk factors, an LDL-C goal of $<2.6 \mathrm{mmol} / \mathrm{L}$ is recommended[26]. In this study, some T2DM patients with moderate risk of ASCVD and LDL-C level $\geqslant 2.6 \mathrm{mmol} / \mathrm{L}$ will also be enrolled. Patients with very-high ASCVD risk are not included in this study, as their LDL-C goal is stricter, less than $1.4 \mathrm{mmol} / \mathrm{L}$ and even lower for example. Under this condition, intensive statins or/and the combination of other cholesterol-lowing drugs should be recommended[27, 28]. Obviously, this situation is not suitable for subjects in this study.

In this study, high $\mathrm{TG}$ level is defined as fasting $\mathrm{TG} \geq 1.7 \mathrm{mmol} / \mathrm{L}$ and $<5.6$ $\mathrm{mmol} / \mathrm{L}$. On the one hand, fasting $\mathrm{TG} \geq 5.6 \mathrm{mmol} / \mathrm{L}$ means high risk of pancreatitis, thus other TG-lowering agents such as fibrates, niacin, and omega-3 fatty acids are needed in addition to statins for diabetic patients with $\mathrm{TG} \geq 5.6 \mathrm{mmol} / \mathrm{L}[29,30]$. To avoid this condition, the subjects with fasting $\mathrm{TG} \geq 5.6 \mathrm{mmol} / \mathrm{L}$ are not enrolled in this study. On the other hand, fasting $\mathrm{TG} \geq 1.7 \mathrm{mmol} / \mathrm{L}$ indicates higher risk of ASCVD although there is no goal of TG in 2019 ESC guidelines[26]. It was showed the combination treatment of statins and fibrates reduced ASCVD events in diabetic patients with fasting TG $\geq 2.3 \mathrm{mmol} / \mathrm{L}[31]$. Recently, high pure eicosapentaenoic acid on the base of statins treatment also had cardiovascular protection in patients with coronary heart disease and elevated TG level(1.52-5.63 $\mathrm{mmol} / \mathrm{L})$, especially in diabetic patients[32]. It supports that diabetic patients with high TG can benefit from TG reduction. Furthermore, ASCVD risk is more closely related to non-fasting TG level than to fasting TG level. It is recommended that non-fasting TG levels in patients whose fasting TG $<1.7 \mathrm{mmol} / \mathrm{L}$ would not exceed $2.0 \mathrm{mmol} / \mathrm{L}(175 \mathrm{mg} / \mathrm{dL})$ after consuming a daily meal[33]. Elevated TG level, especially in the postprandial state, means an increase in remnant lipoproteins in the blood, which is as atherogenic as $\operatorname{LDL}[34,35]$. In this study, both fasting and non-fasting TG levels will be 
1 evaluated after 6-week treatment of Xuezhikang and pravastatin.

Lipoprotein(a), similar in structure to LDL, is a special atherogenic lipoprotein, and high lipoprotein(a) level is closely related to increased residual ASCVD risk[36, 37]. The level of lipoprotein(a) is mainly determined by genetic factors[38]. And it is hardly affected by statins treatment. However, it was found that the fasting lipoprotein(a) level decreased by $23 \%$ after a 6-week Xuezhikang treatment in patients with coronary heart disease[39]. Therefore, this study plans to compare the effects of Xuezhikang and pravastatin on the lipoprotein(a) in T2DM patients with moderate to high risk of ASCVD.

In conclusion, we will evaluate the effect of 6-week treatment of Xuezhikang 1.2 $\mathrm{g} / \mathrm{d}$ and pravastatin $20 \mathrm{mg} / \mathrm{d}$ on the TG level and other blood lipids in patients with T2DM and dyslipidemia, which may provide a more optimized schedule for lipid control in patients with diabetes and dyslipidemia with moderate to high risk of ASCVD.

\section{Ethics and Dissemination}

\section{Ethics approval and consent to participate}

This study was approved by the Ethics Committee of the Second Xiangya Hospital of Central South University and written informed consent will be obtained from all study participants before enrollment.

\section{Availability of data and materials}

The data during the current study are available from the corresponding author on reasonable request.

\section{Competing interests}

The authors declare that they have no competing interests.

\section{Funding}

This study received funding from AstraZeneca Investment(China) Co., Ltd (No. 
1 ESR-20-20663). The funder was not involved in the study design, collection, analysis,

2 interpretation of data, the writing of this article or the decision to submit it for

3 publication.

\section{Authors' contributions}

5 Ling Liu is the primary investigator and designer of this study. Jin Xu, Liling Guo,

6 Liyuan Zhu, Yangrong Tan, Yan Fu, Yingying Xie, Miao Zhang, Tie Wen participated

7 in the design of this study. Jin Xu, Xiao Du, Shilan Zhang, Qunyan Xiang drafted the

8 manuscript. Jin $\mathrm{Xu}$ and Ling Liu revised the manuscript. All authors contributed to the

9 article and approved the submitted version. All authors have accepted responsibility

10 for the entire content of this manuscript and approved its submission.

11 Acknowledgements

12 We thank Dr. Xianghang Luo, Dr. Lin Yang and Dr. Ping Jin for their contribution to

13 the design of this study. 


\section{Reference:}

1. Expert Panel on Detection, E. and A. Treatment of High Blood Cholesterol in, Executive Summary of The Third Report of The National Cholesterol Education Program (NCEP) Expert Panel on Detection, Evaluation, And Treatment of High Blood Cholesterol In Adults (Adult Treatment Panel III). JAMA, 2001. 285(19): p. 2486-97.

2. Cosentino, F., P.J. Grant, V. Aboyans, C.J. Bailey, A. Ceriello, V. Delgado, M. Federici, G. Filippatos, D.E. Grobbee, T.B. Hansen, H.V. Huikuri, I. Johansson, P. Juni, M. Lettino, N. Marx, L.G. Mellbin, C.J. Ostgren, B. Rocca, M. Roffi, N. Sattar, P.M. Seferovic, M. Sousa-Uva, P. Valensi, D.C. Wheeler, and E.S.C.S.D. Group, 2019 ESC Guidelines on diabetes, pre-diabetes, and cardiovascular diseases developed in collaboration with the EASD. Eur Heart J, 2020. 41(2): p. 255-323.

3. Athyros, V.G., M. Doumas, K.P. Imprialos, K. Stavropoulos, E. Georgianou, A. Katsimardou, and A. Karagiannis, Diabetes and lipid metabolism. Hormones (Athens), 2018. 17(1): p. 61-67.

4. Chait, A., H.N. Ginsberg, T. Vaisar, J.W. Heinecke, I.J. Goldberg, and K.E. Bornfeldt, Remnants of the Triglyceride-Rich Lipoproteins, Diabetes, and Cardiovascular Disease. Diabetes, 2020. 69(4): p. 508-516.

5. Toth, P.P., Triglyceride-rich lipoproteins as a causal factor for cardiovascular disease. Vasc Health Risk Manag, 2016. 12: p. 171-83.

6. Verbeek, R., G.K. Hovingh, and S.M. Boekholdt, Non-high-density lipoprotein cholesterol: current status as cardiovascular marker. Curr Opin Lipidol, 2015. 26(6): p. 502-10.

7. Anderson, T.J., J. Gregoire, G.J. Pearson, A.R. Barry, P. Couture, M. Dawes, G.A. Francis, J. Genest, Jr., S. Grover, M. Gupta, R.A. Hegele, D.C. Lau, L.A. Leiter, E. Lonn, G.B. Mancini, R. McPherson, D. Ngui, P. Poirier, J.L. Sievenpiper, J.A. Stone, G. Thanassoulis, and R. Ward, 2016 Canadian Cardiovascular Society Guidelines for the Management of Dyslipidemia for the Prevention of Cardiovascular Disease in the Adult. Can J Cardiol, 2016. 32(11): p. 1263-1282.

8. Stone, N.J., J.G. Robinson, A.H. Lichtenstein, C.N. Bairey Merz, C.B. Blum, R.H. Eckel, A.C. Goldberg, D. Gordon, D. Levy, D.M. Lloyd-Jones, P. McBride, J.S. Schwartz, S.T. Shero, S.C. Smith, Jr., K. Watson, P.W. Wilson, and G. American College of Cardiology/American Heart Association Task Force on Practice, 2013 ACC/AHA guideline on the treatment of blood cholesterol to reduce atherosclerotic cardiovascular risk in adults: a report of the American College of Cardiology/American Heart Association Task Force on Practice Guidelines. J Am Coll Cardiol, 2014. 63(25 Pt B): p. 2889-934.

9. Krahenbuhl, S., I. Pavik-Mezzour, and A. von Eckardstein, Unmet Needs in LDL-C Lowering: When Statins Won't Do! Drugs, 2016. 76(12): p. 1175-90.

10. Ballantyne, C.M., J.S. Raichlen, S.J. Nicholls, R. Erbel, J.C. Tardif, S.J. Brener, V.A. Cain, S.E. Nissen, and A. Investigators, Effect of rosuvastatin therapy on coronary artery stenoses assessed by quantitative coronary angiography: a study to evaluate the effect of rosuvastatin on intravascular ultrasound-derived coronary atheroma burden. Circulation, 2008. 117(19): p. 2458-66.

11. Hunninghake, D.B., E.A. Stein, H.E. Bays, D.J. Rader, R.R. Chitra, S.G. Simonson, and D.W. Schneck, Rosuvastatin improves the atherogenic and atheroprotective lipid profiles in patients with hypertriglyceridemia. Coron Artery Dis, 2004. 15(2): p. 115-23.

12. Roth, E.M., J.M. McKenney, M.T. Kelly, C.M. Setze, D.M. Carlson, A. Gold, J.C. Stolzenbach, L.A. Williams, and P.H. Jones, Efficacy and safety of rosuvastatin and fenofibric acid combination 
therapy versus simvastatin monotherapy in patients with hypercholesterolemia and hypertriglyceridemia: a randomized, double-blind study. Am J Cardiovasc Drugs, 2010. 10(3): p. $175-86$.

13. Farnier, M., J. Ducobu, and L. Bryniarski, Efficacy and safety of adding fenofibrate $160 \mathrm{mg}$ in high-risk patients with mixed hyperlipidemia not controlled by pravastatin $40 \mathrm{mg}$ monotherapy. Am J Cardiol, 2010. 106(6): p. 787-92.

14. Zhao, S.P., R. Li, W. Dai, B.L. Yu, L.Z. Chen, and X.S. Huang, Xuezhikang contributes to greater triglyceride reduction than simvastatin in hypertriglyceridemia rats by up-regulating apolipoprotein A5 via the PPARalpha signaling pathway. PLoS One, 2017. 12(9): p. e0184949.

15. Zhao, S.P., L. Liu, Y.C. Cheng, M.H. Shishehbor, M.H. Liu, D.Q. Peng, and Y.L. Li, Xuezhikang, an extract of cholestin, protects endothelial function through antiinflammatory and lipid-lowering mechanisms in patients with coronary heart disease. Circulation, 2004. 110(8): p. 915-20.

16. Cicero, A.F.G., F. Fogacci, and M. Banach, Red Yeast Rice for Hypercholesterolemia. Methodist Debakey Cardiovasc J, 2019. 15(3): p. 192-199.

17. Jones, P.H., M.H. Davidson, E.A. Stein, H.E. Bays, J.M. McKenney, E. Miller, V.A. Cain, J.W. Blasetto, and S.S. Group, Comparison of the efficacy and safety of rosuvastatin versus atorvastatin, simvastatin, and pravastatin across doses (STELLAR* Trial). Am J Cardiol, 2003. 92(2): p. 152-60.

18. Feillet, C., M. Farnier, L.H. Monnier, C. Percheron, C. Colette, B. Descomps, and A. Crastes De Paulet, Comparative effects of simvastatin and pravastatin on cholesterol synthesis in patients with primary hypercholesterolemia. Atherosclerosis, 1995. 118(2): p. 251-8.

19. Moriarty, P.M., E.M. Roth, A. Karns, P. Ye, S.P. Zhao, Y. Liao, D.M. Capuzzi, H.E. Bays, F. Zhang, S. Liu, A.J. Reichman, O.A. Brusco, G. Lu, S. Lerman, Z. Duan, S. Guo, P.L. Liu, J. Zhao, Y. Zhang, and S. Li, Effects of Xuezhikang in patients with dyslipidemia: a multicenter, randomized, placebo-controlled study. J Clin Lipidol, 2014. 8(6): p. 568-575.

20. Cheng, Y.C., Clinical observation on effect of combination ezetimibe with Xuezhikang on cholestin extract. Journal of Qiqihar University of Medicine, 2014. 35(16): p. 2397-2399.

21. American Diabetes, A., 2. Classification and Diagnosis of Diabetes: Standards of Medical Care in Diabetes-2020. Diabetes Care, 2020. 43(Suppl 1): p. S14-S31.

22. Zhao, S.P., L. Liu, Y.C. Cheng, and Y.L. Li, Effect of xuezhikang, a cholestin extract, on reflecting postprandial triglyceridemia after a high-fat meal in patients with coronary heart disease. Atherosclerosis, 2003. 168(2): p. 375-80.

23. Li, J.J., Z.L. Lu, W.R. Kou, Z. Chen, Y.F. Wu, X.H. Yu, Y.C. Zhao, and G. Chinese Coronary Secondary Prevention Study, Beneficial impact of Xuezhikang on cardiovascular events and mortality in elderly hypertensive patients with previous myocardial infarction from the China Coronary Secondary Prevention Study (CCSPS). J Clin Pharmacol, 2009. 49(8): p. 947-56.

24. Lu, Z., W. Kou, B. Du, Y. Wu, S. Zhao, O.A. Brusco, J.M. Morgan, D.M. Capuzzi, G. Chinese Coronary Secondary Prevention Study, and S. Li, Effect of Xuezhikang, an extract from red yeast Chinese rice, on coronary events in a Chinese population with previous myocardial infarction. Am J Cardiol, 2008. 101(12): p. 1689-93.

25. Zhao, S.P., Z.L. Lu, B.M. Du, Z. Chen, Y.F. Wu, X.H. Yu, Y.C. Zhao, L. Liu, H.J. Ye, Z.H. Wu, and S. China Coronary Secondary Prevention, Xuezhikang, an extract of cholestin, reduces cardiovascular events in type 2 diabetes patients with coronary heart disease: subgroup 
analysis of patients with type 2 diabetes from China coronary secondary prevention study (CCSPS). J Cardiovasc Pharmacol, 2007. 49(2): p. 81-4.

26. Mach, F., C. Baigent, A.L. Catapano, K.C. Koskinas, M. Casula, L. Badimon, M.J. Chapman, G.G. De Backer, V. Delgado, B.A. Ference, I.M. Graham, A. Halliday, U. Landmesser, B. Mihaylova, T.R. Pedersen, G. Riccardi, D.J. Richter, M.S. Sabatine, M.R. Taskinen, L. Tokgozoglu, O. Wiklund, and E.S.C.S.D. Group, 2019 ESC/EAS Guidelines for the management of dyslipidaemias: lipid modification to reduce cardiovascular risk. Eur Heart J, 2020. 41(1): p. 111-188.

27. Handelsman, Y., P.S. Jellinger, C.K. Guerin, Z.T. Bloomgarden, E.A. Brinton, M.J. Budoff, M.H. Davidson, D. Einhorn, S. Fazio, V.A. Fonseca, A.J. Garber, G. Grunberger, R.M. Krauss, J.I. Mechanick, P.D. Rosenblit, D.A. Smith, and K.L. Wyne, Consensus Statement by the American Association of Clinical Endocrinologists and American College of Endocrinology on the Management of Dyslipidemia and Prevention of Cardiovascular Disease Algorithm - 2020 Executive Summary. Endocr Pract, 2020. 26(10): p. 1196-1224.

28. Atherosclerosis, C. Coronary Heart Disease Working Group of Chinese Society of, and C. Editorial Board of Chinese Journal of, [Chinese expert consensus on lipid management of very high-risk atherosclerotic cardiovascular disease patients]. Zhonghua Xin Xue Guan Bing Za Zhi, 2020. 48(4): p. 280-286.

29. Rawla, P., T. Sunkara, K.C. Thandra, and V. Gaduputi, Hypertriglyceridemia-induced pancreatitis: updated review of current treatment and preventive strategies. Clin J Gastroenterol, 2018. 11(6): p. 441-448.

30. Tsuang, W., U. Navaneethan, L. Ruiz, J.B. Palascak, and A. Gelrud, Hypertriglyceridemic pancreatitis: presentation and management. Am J Gastroenterol, 2009. 104(4): p. 984-91.

31. Elam, M.B., H.N. Ginsberg, L.C. Lovato, M. Corson, J. Largay, L.A. Leiter, C. Lopez, P.J. O'Connor, M.E. Sweeney, D. Weiss, W.T. Friedewald, J.B. Buse, H.C. Gerstein, J. Probstfield, R. Grimm, F. Ismail-Beigi, D.C. Goff, Jr., J.L. Fleg, Y. Rosenberg, R.P. Byington, and A.S. Investigators, Association of Fenofibrate Therapy With Long-term Cardiovascular Risk in Statin-Treated Patients With Type 2 Diabetes. JAMA Cardiol, 2017. 2(4): p. 370-380.

32. Bhatt, D.L., P.G. Steg, M. Miller, E.A. Brinton, T.A. Jacobson, S.B. Ketchum, R.T. Doyle, Jr., R.A. Juliano, L. Jiao, C. Granowitz, J.C. Tardif, C.M. Ballantyne, and R.-I. Investigators, Cardiovascular Risk Reduction with Icosapent Ethyl for Hypertriglyceridemia. N Engl J Med, 2019. 380(1): p. 11-22.

33. Nordestgaard, B.G., A. Langsted, S. Mora, G. Kolovou, H. Baum, E. Bruckert, G.F. Watts, G. Sypniewska, O. Wiklund, J. Boren, M.J. Chapman, C. Cobbaert, O.S. Descamps, A. von Eckardstein, P.R. Kamstrup, K. Pulkki, F. Kronenberg, A.T. Remaley, N. Rifai, E. Ros, M. Langlois, S. European Atherosclerosis, C. the European Federation of Clinical, and i. Laboratory Medicine joint consensus, Fasting is not routinely required for determination of a lipid profile: clinical and laboratory implications including flagging at desirable concentration cut-points-a joint consensus statement from the European Atherosclerosis Society and European Federation of Clinical Chemistry and Laboratory Medicine. Eur Heart J, 2016. 37(25): p. 1944-58.

34. Ginsberg, H.N. and D.R. Illingworth, Postprandial dyslipidemia: an atherogenic disorder common in patients with diabetes mellitus. Am J Cardiol, 2001. 88(6A): p. 9H-15H.

35. Bansal, S., J.E. Buring, N. Rifai, S. Mora, F.M. Sacks, and P.M. Ridker, Fasting compared with 
nonfasting triglycerides and risk of cardiovascular events in women. JAMA, 2007. 298(3): p. 309-16.

36. Tada, H., M. Takamura, and M.A. Kawashiri, Lipoprotein(a) as an Old and New Causal Risk Factor of Atherosclerotic Cardiovascular Disease. J Atheroscler Thromb, 2019. 26(7): p. 583-591.

37. Wu, M.F., K.Z. Xu, Y.G. Guo, J. Yu, Y. Wu, and L.M. Lin, Lipoprotein(a) and Atherosclerotic Cardiovascular Disease: Current Understanding and Future Perspectives. Cardiovasc Drugs Ther, 2019. 33(6): p. 739-748.

38. Tsimikas, S., A Test in Context: Lipoprotein(a): Diagnosis, Prognosis, Controversies, and 39. Liu, L., S.P. Zhao, Y.C. Cheng, and Y.L. Li, Xuezhikang decreases serum lipoprotein(a) and C-reactive protein concentrations in patients with coronary heart disease. Clin Chem, 2003. 


\begin{tabular}{|c|c|c|c|c|}
\hline Visits & $\begin{array}{c}\text { Visit } \\
1\end{array}$ & $\begin{array}{c}\text { Visit } \\
2\end{array}$ & $\begin{array}{c}\text { Visit } \\
3\end{array}$ & $\begin{array}{c}\text { Visit } \\
4\end{array}$ \\
\hline Time point(d) & -14 & -1 & 0 & 42 \\
\hline Informed Consent & $x$ & & & \\
\hline Inclusion/Exclusion criteria & $x$ & $x$ & & \\
\hline Randomization & & & $\times$ & \\
\hline Dietary guidance & $x$ & $x$ & $x$ & $x$ \\
\hline Demographic data & $\times$ & & & \\
\hline Anamnesis & $\times$ & $\times$ & & \\
\hline Physical examination & $\times$ & $\times$ & $\times$ & $\times$ \\
\hline Nutritious breakfast & & & $x$ & $\times$ \\
\hline Fasting blood lipid routine (TC、TG、HDL-C、LDL-C) & & $\times$ & & \\
\hline Fasting HbA1c\% levels & & $x$ & & $\times$ \\
\hline $\begin{array}{l}\text { Blood lipids complete set (TC、TG、HDL-C、LDL-C , nonHDL-C、 } \\
\text { RC、 Lp (a) 、Apo (A) 、ApoB ) at fasting and } 2,4 \mathrm{~h} \text { after the meal, } \\
\text { blood lipid routine (TC、TG、HDL-C、LDL-C) at } 1 \mathrm{~h} \text { after the meal }\end{array}$ & & & $x$ & $x$ \\
\hline $\begin{array}{l}\text { hypersensitive C-reactive protein(hs-CRP) at fasting and 2,4 h } \\
\text { after the meal }\end{array}$ & & & $x$ & $x$ \\
\hline Insulin levels at fasting and $1,2 \mathrm{~h}$ after the meal & & & $\times$ & $\times$ \\
\hline Plasma Glucose (PG) levels at fasting and 1,2 $\mathrm{h}$ after the meal & & & $\times$ & $\times$ \\
\hline $\begin{array}{l}\text { blood routine, urine routine, urine pregnancy test (for fertile } \\
\text { women) }\end{array}$ & & $x$ & & $x$ \\
\hline Creatine kinase $(\mathrm{CK})$ & & $\times$ & & $\times$ \\
\hline Hepatic function test & & $\times$ & & $\times$ \\
\hline Renal function test & & $\times$ & & $\times$ \\
\hline ECG & & $\times$ & & $\times$ \\
\hline Medication guidance & & & $\times$ & \\
\hline Record medication & & & & $\times$ \\
\hline Record adverse events & $\times$ & $x$ & $\times$ & $\times$ \\
\hline Record combined medication & $\times$ & $x$ & & $\times$ \\
\hline $\begin{array}{l}\text { Distributing a snack containing carbohydrate before leaving } \\
\text { hospital }\end{array}$ & & & $x$ & $x$ \\
\hline Distributiing drugs and log cards & & & $\times$ & \\
\hline Retrieving drugs and log cards & & & & $x$ \\
\hline
\end{tabular}


Figures

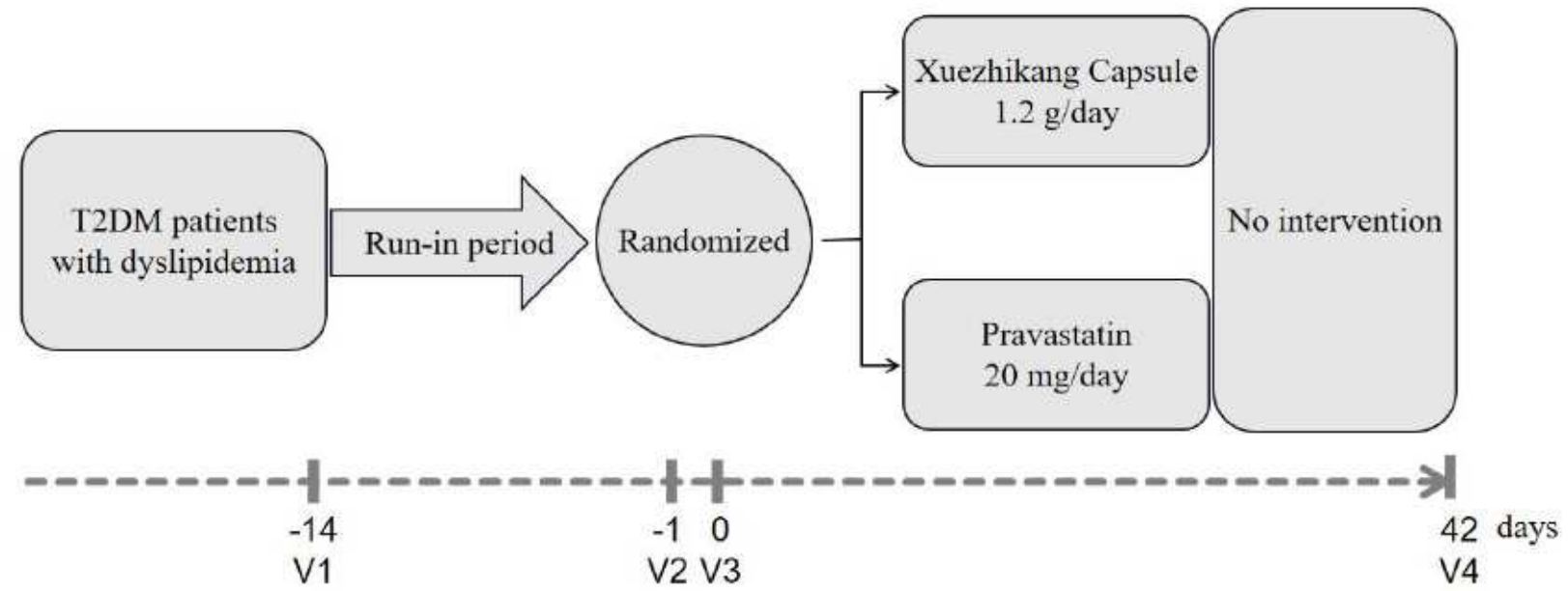

Figure 1

Study design. 